\title{
Applications of Bootstrap in Analyzing General Extreme Value Distributions
}

\author{
Dang Kien Cuong ${ }^{1}$, Duong Ton Dam², Duong Ton Thai Duong ${ }^{3}$ and Ngo Thuan $\mathrm{Du}^{4}$ \\ 1. Information Technology, Nong Lam University, Ho Chi Minh City, Vietnam \\ 2. University of Information Technology, Vietnam National University Ho Chi Minh City, Vietnam \\ 3. Vietnam National University of Ho Chi Minh City, Vietnam \\ 4. CanTho University, Vietnam
}

\begin{abstract}
The bootstrap method is one of the new ways of studying statistical math which this article uses but is a major tool for studying and evaluating the values of parameters in probability distribution. Our research is concerned overview of the theory of infinite distribution functions. The tool to deal with the problems raised in the paper is the mathematical methods of random analysis (theory of random process and multivariate statistics). In this article, we introduce the new function to find out the bias and standard error with jackknife method for Generalized Extreme Value distributions.
\end{abstract}

Key words: Bootstrap method, time series, block bootstrap jackknife method, generalized extreme value distributions.

\section{Basic Framework}

\subsection{Block Bootstrap Methods for Time Series}

Let $X_{1}, X_{2}, \ldots, X_{n}$ be independent and identically random variables with distribution function $F$. The first step in extreme value theory is to investigate the distribution of $M_{n}=\max \left(X_{1}, X_{2}, \ldots, X_{n}\right)$ as $n \rightarrow \infty$. Suppose having sequences of constants $a_{n}>0, b_{n} \in R$ such that:

$$
\begin{gathered}
P\left(M_{n} \leq a_{n} x+b_{n}\right)= \\
\lim _{n \rightarrow \infty} \lim F^{n}\left(a_{n} x+b_{n}\right)=G(x), \forall x \in C(G)
\end{gathered}
$$

where $G(x)$ is a non-degenerate distribution function, $C(G)$ is the set of all continuity points of $G(x)$. Limit distribution functions $G(x)$ satisfying Eq. (1) are the well known extreme value three types of distributions (Frechet, Weibull, and Gumbel distributions).

The generalized extreme value (GEV) family of distribution is:

Corresponding author: Dang Kien Cuong, Ph.D. student, lecture, research fields: data science, business intelligence, data mining, education management, machine learning.

$$
G(X)=e^{-\left(1+\xi\left(\frac{x-\mu}{\sigma}\right)\right)^{-\frac{1}{\xi}}},\left\{x: 1+\xi\left(\frac{x-\mu}{\sigma}\right)>0\right\}
$$

where $\mu$ is a location parameter $(\mu \in R), \sigma$ is a scale parameter $(\sigma>0)$, and $\xi$ is the extreme value shape parameter.

Observations $X_{1}, X_{2}, \ldots, X_{n}$ (realisations of a stationary process) are not independent, dependence in time series is relatively simple example of dependent data. Block bootstrap methods for time series data and spatial data have been put forward by Efron, Hall, Radovanov, B., and Marcikie A in Refs. [7, 9-10] among others. See four types of bootstrap methods.

- Moving Block Bootstrap (MBB)

Blocks length $l$, starting at $X_{i}$ : $B_{i}=\left(X_{i}, X_{i+1}, \ldots, X_{i+l-1}\right)$. To get a bootstrap sample:

- Draw with replacement $B_{1}^{*}, B_{1+1}^{*}, \ldots, B_{k}^{*}$ from $B_{1}, B_{2}, \ldots, B_{n-l+1}$.

- Concatenate the blocks $B_{1}^{*}, B_{2}^{*}, \ldots, B_{k}^{*}$ to give the bootstrap sample $X_{1}^{*}, X_{2}^{*}, \ldots, X_{k l}^{*}$.

$-l=1$, corresponds to the classical i.i.d bootstrap.

- Blocks in the MBB may overlap.

- Non-overlapping Block Bootstrap (NBB)

Blocks of length $l: B_{1}=\left(X_{1}, X_{2}, \ldots, X_{l}\right) ; B_{2}=$ 
$\left(X_{l}, X_{l+1}, \ldots, X_{2 l}\right) \ldots$

$$
B_{\left[\frac{n}{l}\right]}=\left(X_{n-l+1}, X_{n-l+2}, \ldots, X_{n}\right) .
$$

- Resample blocks $\mathrm{B}_{1}^{*}, \mathrm{~B}_{2}^{*}, \ldots, \mathrm{B}_{\left[\frac{\mathrm{n}}{1}\right]}^{*} \quad$ with replacement, concantenate to get bootstrap sample $\left(\mathrm{X}_{1}^{*}, \mathrm{X}_{2}^{*}, \ldots, \mathrm{X}_{\left[\frac{n}{l}\right]}^{*}\right)$.

$-b=\left[\frac{n}{l}\right]$ blocks $\left(\left[\frac{n}{l}\right]\right.$ is the largest integer less than or equal to $\frac{n}{l}$ ).

$$
B_{i}=\left(X_{i l}, X_{i l+1}, \ldots, X_{i l+l-1}\right), i=1,2, \ldots,\left[\frac{n}{l}\right] .
$$

- Note: NBB fewer blocks than in the MBB.

- Circular block bootstrap (CBB)

Blocks of length $l$ : Sample with replacement from $\left\{B_{1}, B_{2}, \ldots, B_{m}\right\}$;

$l b=m \approx n$, every observation reseives equal weight:

$B_{i}=\left(X_{i}, X_{i+1}, \ldots, X_{i+l-1}\right) ; i=1,2, \ldots, m$.

- Stationary block bootstrap (SB)

- Blocks are no longer of equal size. The bootstrap sample is chosen according to some probability measures on the sequences. A stationary bootstrap method involves sampling blocks of random length of each block that has a geometric distribution:

$\left(H_{1}, K_{1}\right),\left(H_{2}, K_{2}\right), \ldots \quad H \sim$ Uniform $(1,2, \ldots, n)$, $K \sim \operatorname{Geometric}(p)$ for some $p>0$.

\subsection{Properties of Block Bootstrap Methods}

Through a simulation study we found that the pseudo data generated from the bootstrap method always showed a weaker dependence among the observations than the time series they were sampled from, hence we can draw the conclusion that even by resampling blocks instead of single observations we will lose some of structural from the original sample.

- The pseudo time series generated by the moving block method is not stationary, even if the original series $X_{t}$ is stationary.
The pseudo time series generated by the stationary bootstrap method is actually a stationary time series.

- The mean $\overline{\mathrm{X}}_{\mathrm{N}}^{*}$ of the moving block boostrap is biased in the sense that:

$$
E\left(\bar{X}_{N}^{*} \mid X_{1}, X_{2}, \ldots, X_{n}\right)-\bar{X}_{n} \neq 0 .
$$

- The MBB estimator of the variance of $\sqrt{n} \bar{X}_{n}$ is also biased. This creates problems in using the percentile-t method with the MBB.

The usual estimator:

$$
\hat{\sigma}^{2}=\frac{1}{n} \sum_{i=1}^{n}\left(X_{i}-\bar{X}_{n}\right)^{2}
$$

should be modified to:

$$
\begin{aligned}
\hat{\sigma}^{2}=\frac{1}{n} \sum_{i=1}^{n}\left\{\left(X_{i}\right.\right. & \left.-\bar{X}_{n}\right)^{2} \\
& \left.+\sum_{k=1}^{i-1} \sum_{i=1}^{n-k}\left(X_{i}-\bar{X}_{n}\right)\left(X_{i+k}-\bar{X}_{n}\right)\right\}
\end{aligned}
$$

With this modification the bootstrap- $t$ can improve substantially on the normal approximation.

- Comparison of block bootstrap methods: We find that overall the MBB and $\mathrm{CBB}$ methods give the bootstrap estimators with the smallest standard error and the SB method the largest (random block length leads to a larger variance of the parameter estimates than for the other methods when block length is fixed). In terms of the bias of the bootstrap estimators there is no method outperforming the other.

\subsection{The Block Bootstrap Procedure}

Assume that the statistic $\hat{\theta}$ estimates a functional $\theta$, depending on the $m$-dimensional marginal distribution of the time series. Now, build vectors of consecutive observations.

$$
Y_{t}=\left(X_{t-m+1}, \ldots, X_{t}\right), \quad t=m, \ldots, n .
$$

Build overlapping block of consecutive vectors, $\left(Y_{m}, \ldots, Y_{m+l-1}\right),\left(Y_{m+1}, \ldots, Y_{m+l}\right), \ldots,\left(Y_{m+l-1}, \ldots, Y_{n}\right)$ where $l \in N$ is the block length parameter. For simplicity, assume first that: 
$m+l-1=k l$ with $k \in N$. Then, resample $k$ block independently,

$Y_{S_{1}+1}, \ldots, Y_{S_{1}+l}, Y_{S_{2}+1}, \ldots, Y_{S_{2}+l}, \ldots, Y_{S_{k}+1}, \ldots, Y_{S_{k}+l}$, where the block starting points $S_{1}, \ldots, S_{k}$, are i.i.d. Uniform $(\{m-1, \ldots, n-l\})$. These resampled block of $m$-vectors could be referred to the block bootstrap sample. However, as we will see, the block bootstrapped estimator is not just simply defined by the plug-in rule and the notion of a bootstrap sample is not clear. If $n-m+1$ is not a multiple of $l$, we resample $k=[(n=m+1) / l]+1$ blocks but use only a portion of the $k$-th block to get $n-m+1$ resampled vectors in total.

$$
\begin{aligned}
& \text { Assume that } \hat{\theta}=T\left(F_{n}^{(m)}\right) \\
& \qquad \begin{aligned}
\hat{\theta}^{* B} & =\varphi_{n-m+1}\left(Y_{S_{1}+1}, \ldots, Y_{S_{1}+l}, Y_{S_{2}+1}, \ldots, Y_{S_{2}+l}, \ldots, Y_{S_{k}+1}, \ldots, Y_{S_{k}+l}\right) \\
\hat{\theta} & =\varphi_{n-m+1}\left(Y_{m}, \ldots, Y_{n}\right),
\end{aligned}
\end{aligned}
$$

saying that it employs a plus-in rule based on vectorized observations.

\subsection{Choosing an Optimal Block Length}

The orders of magnitude of the optimal block size are known in some inference problems [3, 8]. According to the those authors three different settings of practical importance can be identified: estimation of the bias or variance, estimation of a one-sided distribution function and estimation of a two-sided distribution function.

The optimal block length in the above situations of different size is $b \sim C n^{\frac{1}{k}}, k=3,4, \ldots$ respectively

This result, will be used here as the basis for the choosing the optimal block length. Two main approaches can be pointed out:

A cross validation method was proposed by Hall et al. [9] and a plug-in method based on a recent work of Castollo et al. [2].

Based on a work of Lahiri, a nonparametric plug-in (NPPI) method for selecting the optimal block length in order to reduce the bias, will be considered. Unlike where $n$ is the sample size. where $F_{n}^{(m)}(x)=\frac{1}{n-m+1} \sum_{i=m}^{n} 1_{\left[Y_{t} \leq x\right]} \quad$ is empirical distribution function of the $m$-dimensional marginal distribution of $\left(X_{t}\right)_{t \in Z}$, and $T$ is smooth functional.

The block bootstrapped estimator is defined as:

$$
\begin{gathered}
\hat{\theta}^{* B}=T\left(F_{n}^{(m) * B}\right) \\
F_{n}^{(m) * B}(x)=\frac{1}{n-m+1} \sum_{i=1}^{k} \sum_{t=S_{i}+1}^{S_{i}+l} 1_{\left[Y_{t} \leq x\right]}
\end{gathered}
$$

This definition of the block bootstrapped estimator, can be interpreted as: we have $E^{* B}\left[\hat{\theta}^{* B}\right] \neq \hat{\theta}$.

traditional plug-in method this method employs nonparametric resampling procedures to estimate the relevant constants in the leading term of the optimal block length.

The variance of block bootstrap estimator is an increasing function of the block length $l$ while its bias is a decreasing function of $l$. As a result, for each block bootstrap estimator, there is critical value, $l_{n}^{0}$, that minimizes mean-square error (MSE). The value of $l$ that minimizes the leading term in the expension of the MSE is denominated MSE-optimal block length.

The following notation will be used:

$$
\begin{aligned}
& \Delta_{n}=\theta\left(m_{n}\right)-\theta(\mu) ; \beta=E \Delta_{n} ; \sigma_{\infty}^{2}=\operatorname{Var}\left(\sqrt{n} \Delta_{n}\right) ; \\
& \widehat{\Delta}_{n}=\theta\left(\widehat{m}_{n}\right)-\theta\left(\hat{E} \widehat{m}_{n}\right) ; \hat{\beta}=\hat{E} \hat{\Delta}_{n} ; \hat{\sigma}_{\infty}^{2}= \\
& \widehat{\operatorname{Var}}\left(\sqrt{n} \hat{\Delta}_{n}\right) .
\end{aligned}
$$

The block bootstrap may use either overlapping or non-overlapping blocks. Define one-sided and symmetrical distribution functions of the normalized statistic $\sqrt{n} \frac{\Delta_{n}}{\sigma_{\infty}}$ by $\quad F_{1}(z)=P\left(\sqrt{n} \frac{\Delta_{n}}{\sigma_{\infty}}<z\right) \quad$ and $F_{2}(z)=P\left(\sqrt{n} \frac{\left|\Delta_{n}\right|}{\sigma_{\infty}} \leq z\right)$.

Define bootstrap analogs of $F_{1}$ and $F_{2}$ by 
$\hat{F}_{1}(z)=\hat{P}\left(\sqrt{n} \frac{\hat{\Delta}_{n}}{\widehat{\sigma}_{\infty}}<z\right)$ and $\hat{F}_{2}(z)=\hat{P}\left(\sqrt{n} \frac{\left|\hat{\Delta}_{n}\right|}{\hat{\sigma}_{\infty}}<z\right)$.

Let $(\psi, \hat{\psi})$ denote either $(\beta, \hat{\beta})$ or $\left(\frac{1}{n} \sigma_{\infty}^{2}, \frac{1}{n} \hat{\sigma}_{\infty}^{2}\right)$, and $\phi$ denote the standard normal density function: $\phi(x)=\frac{1}{\sqrt{2 \pi}} \exp \left\{-\frac{1}{2} x^{2}\right\}$.

Hall [9] shows that there are constants $C_{i}(i=1$, $2, \ldots, 6$ such that, in addition, $n-1+n-1 l=o 1$ as $n \rightarrow \infty$, then:

$$
\begin{gathered}
E(\psi-\hat{\psi})^{2} \sim \frac{1}{n^{2}}\left(C_{1} \frac{1}{l^{2}}+C_{2} \frac{1}{l}\right), \\
E\left[F_{1}(z)-\hat{F}_{1}(z)\right]^{2} \sim \frac{1}{n}\left(C_{3} \frac{1}{l^{2}}+C_{4} \frac{l^{2}}{n}\right) \phi(z)^{2}, \\
E\left[F_{2}(z)-\hat{F}_{2}(z)\right]^{2} \sim \frac{1}{n^{2}}\left(C_{5} \frac{1}{l^{2}}+C_{6} \frac{l^{3}}{n}\right) \phi(z)^{2},
\end{gathered}
$$

where the symbol $\sim$ indicates that the quantiny on the right-hand side is the leading term of an asymptotic expansion. The constants $C_{i}$ do not depend on $n$ or $l$. The terms involving $C_{2}, C_{4}$ and $C_{6}$ correspond to the variance. The variance terms are smaller if the bocks are overlapping than if they are non-overlapping.

It follows from $\left({ }^{*}\right),\left({ }^{* *}\right),\left({ }^{* * *}\right)$ that the asymptotically optimal block length (in the sense of minimizing the AMSE) is $l=A_{1} n^{1 / 3}$ for bias or variance estimation, $l=A_{2} n^{1 / 4}$ for estimating a one-sided distribution function, and $l=A_{3} n^{1 / 5}$ for estimating a symmetrical distribution function ( $A_{j}>0 ; j=1,2,3$, are suitable constants that depend on certain population parametrs).

\section{Jackknife Method}

We assume a vector of parameters such as $\theta$. The bias of $\theta$ as an estimate of an estimator $\hat{\theta}_{0}$ of $\theta_{0}$ is defined by $\Delta=E \hat{\theta}_{0}-\theta_{0}$.

A large bias is usually an undesirable aspect of an estimator performance. We can use the bootstrap to assess the bias of any estimator $\theta$ as an estimate of an estimator $\hat{\theta}_{0}$. We generate $B$ independent bootstrap samples $X^{*}, X^{*^{2}}, \ldots, X^{*^{B}}$, each consisting of $n$ data value drawn with replication corresponding to each bootstrap sample from $X$, as $X^{*^{1}}=X_{i 1}, X^{*^{2}}=X_{i 2}, \ldots$, $X^{*^{n}}=X_{\text {in }}$.

We can select the sample of $B$ in the range 25-1,000. Then the bootstrap application is evaluated corresponding to each bootstrap sample, it may be an indication that the statistic $\hat{X}^{*}(b)=S X^{* b}, b=1$, $2, \ldots B$. The bootstrap estimate of bias is defined by $\Delta_{B}=\hat{\theta}_{0}^{*}-\hat{\theta}_{0}$ where $\hat{\theta}_{0}^{*}=\frac{1}{n} \sum_{b=1}^{B} \hat{\theta}_{b}^{*}$.

We have concentrated on standard error as a measure of accuracy for an estimator $\hat{\theta}_{0}$. Estimate the standard error $s e_{B}(\hat{\theta})$ by the sample standard deviation of the $B$ replications,

$$
\widehat{s e}_{B}=\left[\frac{1}{B-1} \sum_{b=1}^{B}\left(\widehat{\theta}^{*}(b)-\widehat{\theta}_{0}^{*}\right)^{2}\right]^{\frac{1}{2}}
$$

The jackknife estimate of bias is another method to find out the bias which it was original computer based method for estimating biases and standard errors. Suppose we have a sample $x=\left(x_{1}, x_{2}, \ldots, x_{n}\right)$ and an estimator $\hat{\theta}_{0}=S(X)$. The $i^{\text {th }}$ jackknife sample $x_{(i)}$, is defined to be $x$ with $i^{\text {th }}$ data point removed, $x_{(i)}=\left(x_{1}, x_{2}, \ldots, x_{i-1}, x_{i+1}, \ldots x_{n}\right)$.

For $i=1,2, \ldots, n$, The jackknife estimate of bias is defined by:

$$
\Delta_{\text {jack }}=(n-1)\left(\hat{\theta}_{(.)}-\hat{\theta}_{0}\right)
$$

where $\hat{\theta}_{(.)}=\frac{1}{n} \sum_{i=1}^{n} \hat{\theta}_{(i)}$. The jackknife estimate of standard error is $\widehat{s e}_{j a c k}=\left[\frac{n-1}{n} \sum_{i=1}^{n}\left(\widehat{\theta}_{(i)}-\hat{\theta}_{(.)}\right)^{2}\right]^{\frac{1}{2}}$.

The jackknife often provides a simple and good approximation to the bootstrap, for estimation of standard error and bias.

As a rule of thumb, a bias of less than 0.25 standard errors can be ignored, unless we are trying to do careful confidence interval calculations. The root mean square error of an estimator $\hat{\theta}$ for $\theta$ is $\sqrt{E(\hat{\theta}-\theta)^{2}}$ a measure of accuracy that takes into account both bias and standard error. It can be shown that the root mean square equals, 


$$
\sqrt{E(\hat{\theta}-\theta)^{2}}=\widehat{s e} \sqrt{1+\left(\frac{\Delta}{\widehat{s e}}\right)^{2}} \cong \widehat{s e}\left[1+0.5\left(\frac{\Delta}{\widehat{s e}}\right)^{2}\right]
$$

If $\Delta=0$ then the root mean square equals its minimum value of standard error. Reverse, rate $\left|\frac{\Delta}{\widehat{s e}}\right|<0.25$, then the root mean square error is no more that about 0.031 greater than value of standard error.

The obvious bias corrected estimator is, $\theta_{\text {corr }}=$ $\hat{\theta}_{0}-\Delta=2 \hat{\theta}_{0}-\hat{\theta}_{0}^{*}$ where, $\Delta=\Delta_{B}$. When bias is small compared to the estimated standard error se; then it is $b$ safer to use $\hat{\theta}_{0}$ than $\theta_{\text {corr }}$. Reverse, bias is large compared to standard error, then it may be an indication that the statistic $\hat{\theta}_{0}=S(X)$ is not an appropriate estimate of the parameter $\theta$.

Quantifying the precision of an estimator can usually be made more explicit by calculating a confidence interval. A standard result says that $\hat{\theta}_{0}$ is the maximum likelihood estimator that has a limiting multivariate normal distribution with mean $\theta_{0}$ and variance covariance matrix $H_{\theta_{0}}=I\left(\theta_{0}\right)^{-1}$, where $I(\theta)=\left[e_{i, j}(\theta)\right]_{d \times d}$ with $e_{i, j}(\theta)=-\mathrm{E} \frac{\partial^{2} \mathrm{~L}(\theta)}{\partial \theta_{\mathrm{i}} \partial \theta_{\mathrm{j}}}$, and $\mathrm{L}(\theta)=\sum_{i=1}^{n} \log f_{0}\left(x_{i}\right)$ is likehood function.

The matrix $I(\theta)$ is "Fischer's information matrix". Since the true value of $\theta_{0}$ is generally unknown, it is usual to approximate the term of $I$ with those of the "Fischer's information matrix" defined by $I_{0}(\theta)=$ $\left[-\frac{\partial^{2} L(\theta)}{\partial \theta_{i} \partial \theta_{j}}\right]_{d \times d}$ and evaluated at $\theta=\hat{\theta}$. Denoting an arbitrary term in the inverse of $I_{O}(\theta)$ by $\tilde{\sigma}_{i, j}$, it follows that an approximate ( $1 \quad \tau$ ) with $0<\tau<1$, confidence interval for $\theta_{i}$ is $\hat{\theta}_{i} \pm Z_{\frac{\tau}{2}} \sqrt{\tilde{\sigma}_{i, j}}$.

Let $\hat{\theta}_{0}$ be the maximum likelihood estimator of the $k$-dimensional parameter $\theta_{0}$ with approximate variance covariance matrix $H_{\theta_{0}}$.

Moreover, a confidence interval can be derived from the likelihood function, by using approximation $\mathcal{L}\left(\theta_{0}\right)=2\left(L\left(\hat{\theta}_{0}\right)-L\left(\theta_{0}\right)\right) \sim \chi_{2}^{2}$.
It follows that an approximate ( $\begin{array}{ll}\tau & \tau) \text { confidence }\end{array}$ region for $\theta_{0}$ is given by $C_{\tau}=\left\{\theta: L(\theta) \leq c_{\tau}\right\}$, where $c_{\tau}$ is the $(1-\tau)$ quantile of the $\chi_{d}^{2}$ distributtion. This approximation is usually more accurate than that based on the asymptotic normality of the maximum likelihood estimator.

The log likelihood for $\theta$ can be formally written as $L_{p}(\theta)=\max L\left(\theta^{(1)}, \theta^{(2)}\right)$ where $\theta$ has two components.

The profile $\log$ likelihood for $\theta^{(1)}$ is now define as:

$$
L_{p}\left(\theta^{(1)}\right)=\max _{\theta^{(2)}} L\left(\theta^{(1)}, \theta^{(2)}\right)
$$

The similarly,

$$
L_{p}\left(\theta^{(2)}\right)=\max _{\theta^{(1)}} L\left(\theta^{(1)}, \theta^{(2)}\right) .
$$

Then, under suitable regularly conditions, for large $n$,

$$
\mathcal{L}_{p}\left(\theta^{(1)}\right)=2\left\{L\left(\hat{\theta}_{0}\right)-L_{p}\left(\theta^{(1)}\right)\right\} \sim \chi_{k}^{2} .
$$

For a single component $\theta_{i}, C_{r}=\left\{\theta_{i}: \mathcal{L}_{p}\left(\theta_{i} \leq c_{r}\right)\right\}$ is a $(1-\tau)$ confidence interval, where $c_{r}$ is the $(1-\tau)$ quantile of the $\chi_{1}^{2}$ distribution.

Another method of model selection is the Akaike Information Criterion (AIC). The AIC has played a significant role in solving problems in a wide variety of fields as a model selection criterion for analyzing actual data. The AIC is defined by AIC = -2 (maximum log likelihood) +2 (number of free parameters).

The number of free parameters in a model refers to the dimensions of the parameter vector $\theta$ contained in the specified model $f(x \mid \theta)$.

\section{Analysis for GEV Distributions}

A potential difficulty with the use of likelihood methods for the GEV concerns the regularity conditions that are required for the usual asymptotic properties associated with the maximum likelihood estimator to be valid. Such conditions are not satisfied by the GEV model because the end points of the GEV distribution are functions of the parameter values, 
$\mu-\frac{\sigma}{\xi}$ is an upper end-point of the distribution when $\xi$ $<0$; and a lower end-point when $\xi>0$. This violation of the usual regularity conditions means that the standard asymptotic likelihood results are not automatically applicable. This problem studied in detail and obtained the following results.

(i) if $\xi>-0.5$ maximum likelihood estimators are regular, in the sense of having the usual, asymptotic properties;

(ii) when $-1<\xi<-0.5$, maximum likelihood estimators are generally obtainable, but do not have the standard asymptotic properties, and;

(iii) with $\xi<-1$, maximum likelihood estimators are unlikely to be obtainable.

Under the assumption that $X_{1}, X_{2}, \ldots, X_{m}$ are independent random variables having the GEV distribution, the log likelihood for the GEV parameters when $\xi \neq 0$ is:

$$
\begin{gathered}
L(x, \mu, \sigma, \xi)=-m \log \sigma-\left(1+\frac{1}{\xi}\right) \sum_{i=1}^{m} \log [1+ \\
\xi x i-\mu \sigma-i=1 m \log 1+\xi x i-\mu \sigma-1 \xi
\end{gathered}
$$

where $1+\xi\left(\frac{x_{i}-\mu}{\sigma}\right)>0$, with $i=1,2, \ldots, m$.

The case $\xi \rightarrow 0$ requires separate treatment using the Gumbel limit of the GEV distribution. This leads to the log likelihood:

$$
\begin{gathered}
L(x, \mu, \sigma, \xi)= \\
-m \log \sigma-\sum_{i=1}^{m}\left(\frac{x_{i}-\mu}{\sigma}\right)-\sum_{i=1}^{m} \exp \left[-\left(\frac{x_{i}-\mu}{\sigma}\right)\right]
\end{gathered}
$$

There is no analytical solution, but for any given dataset the maximization is straightforward using standard numerical optimization algorithms.

Estimates of extreme quantile of the maximum distribution under linear normalization are obtained by inverting Eq. (1).

$$
x_{p}= \begin{cases}\mu-\frac{\sigma}{\xi}\left(1-(-\log (1-p))^{-\xi}\right) ; & \xi \neq 0 \\ \mu-\sigma \log (1-p) ; & \xi=0\end{cases}
$$

The return levels are exceeded by the annual maximum in any particular time with probability $\left(\begin{array}{ll}1 & p\end{array}\right)$. If $x_{p}$ are plotted against $\frac{1}{1-p}$ the plots are linear. By substituting the maximum likelihood estimates of the GEV parameters into Eq. (7), the maximum likelihood estimate of $x_{p}$ for $0<p<1$, is obtained as:

$$
\hat{x}_{p}=\left\{\begin{array}{l}
\hat{\mu}-\frac{\hat{\sigma}}{\hat{\xi}}\left(1-y_{p}^{-\xi}\right), \hat{\xi} \neq 0 \\
\hat{\mu}-\hat{\sigma} \log \left(y_{p}\right), \hat{\xi}=0
\end{array}\right.
$$

where $y_{p}=-\log (1-p)$.

By the delta method, we get:

$$
\operatorname{Var}\left(x_{p}\right) \cong \nabla x_{p}^{T} H_{0} \nabla x_{p} \text {, }
$$

where $\theta=[\mu, \sigma, \xi], H_{0}$ is variance covariance matrix, and:

$$
\begin{aligned}
\nabla x_{p}^{T}=\left[\frac{\partial x_{p}}{\partial \mu}, \frac{\partial x_{p}}{\partial \sigma}\right. & \left., \frac{\partial x_{p}}{\partial \xi}\right] \\
= & {\left[1,-\xi^{-1}\left(1-y_{p}^{-\xi}\right), \sigma \xi^{-2}(1\right.} \\
& \left.\left.-y_{p}^{-\xi}\right)-\sigma \xi^{-1} y_{p}^{-\xi} \log \left(y_{p}\right)\right]
\end{aligned}
$$

corresponding value is $(\widehat{\mu}, \widehat{\sigma}, \widehat{\xi})$.

When the $\hat{\xi} \rightarrow 0$ and Eq. (9) is still valid with:

$$
\nabla x_{p}^{T}=\left[1, \log y_{p}\right]
$$

corresponding value is $(\widehat{\mu}, \widehat{\sigma})$.

\subsection{Profile Likelihood}

Numerical evaluation of the profile likelihood for any of the individual parameters $\mu, \sigma$ or $\xi$ is straightforward. For example, to obtain the profile likelihood for $\xi$, we fix $\xi=\xi_{0}$, and maximize the log likelihood (5) with respect to the remaining parameters, $\mu$ and $\sigma$. This is repeated for a range of values of $\xi_{0}$. This methodology can also be applied when inference is required on some combination of parameters. In particular, we can obtain the profile likelihood for any specified return level $x_{p}$ : This requires a re-parameterization of the GEV model, so that $x_{p}$ is one of the model parameters, after which the profile log likelihood is obtained by maximization with respect to the remaining parameters in the usual way. Re-parameterization is straightforward, 


$$
\mu= \begin{cases}x_{\boldsymbol{p}}-\frac{\sigma}{\xi}\left(1-y_{p}^{-\xi}\right), & \xi \neq 0, \\ x_{\boldsymbol{p}}-\sigma \log \left(y_{\boldsymbol{p}}\right), & \xi=0 .\end{cases}
$$

so that replacement of $\mu$ in Eqs. (5), (6) with (11) has the desired effect of expressing the GEV model in terms of the parameters $\left(x_{p}, \sigma, \xi\right)$.

\subsection{Model Validity}

A probability plot is a comparison of the empirical and fitted distribution functions. With ordered block maximum data $x_{1} \leq x_{2} \leq \ldots \leq x_{m}$, the empirical distribution function evaluated at $x_{i}$ is given by $\check{G}\left(x_{(i)}\right)=\frac{i}{m+1}$.

By substitution of parameter estimates into Eq. (2), the corresponding model based estimates is:

$$
\begin{aligned}
& \widehat{G}\left(x_{(i)}\right) \\
& = \begin{cases}\exp \left[-\left(1+\hat{\xi}\left(\frac{x_{(i)}-\widehat{\mu},}{\hat{\sigma}}\right)\right)^{-\frac{1}{\xi}}\right] ; & \xi \neq 0, \\
\exp \left[-\exp \left(-\left(\frac{x_{(i)}-\widehat{\mu}_{,}}{\hat{\sigma}}\right)\right)\right] ; & \xi \neq 0 .\end{cases}
\end{aligned}
$$

We then construct plot consisting of the points.

$$
\left\{\left(\check{G}^{-1}\left(x_{(i)}\right), \hat{G}\left(x_{(i)}\right)\right), i=1,2, \ldots, m\right\}
$$

A weakness of the probability plot for extreme value models is that both $\breve{G}^{-1}\left(x_{(i)}\right)$ and $\hat{G}\left(x_{(i)}\right)$ are bound to approach 1 as $x_{(i)}$ increases, while it is usually the accuracy of the model for large values of $x$ that is of greatest concern. That is, the probability plot provides the least information in the region of most interest. This deficiency is avoided by the quantile plot, consisting of the points.

$$
\left\{\left(\hat{G}^{-1}\left(\frac{i}{m+1}\right), x_{(i)}\right), i=1,2, \ldots, m\right\}
$$

If $\hat{G}$ is a reasonable estimate of $G$, then the quantile plot should also consist of points close to the unit diagonal.

\section{Conclusion}

To estimate the value of a parameter in GEV we can use classical methods of mathematical statistics such as the maximum likelihood method or the least squares method but they all require a certain number, samples for verification. For the bootstrap method, this is obviously not needed, here we use the limit theorems of probability theory and multivariate statistics to solve the problem even if there is only one sample data. That is the important practical significance that our paper wants to convey.

\section{Acknowledgment}

This research is funded by Vietnam National University Ho Chi Minh City (VNU-HCM) under grant number C2017-26-03.

\section{References}

[1] Coles, S. 2001. An Introduction Statistical Modeling of Extreme Values. Springer.

[2] Castollo, E., Hadi, A. S., Balakrishnan, N., and Sarabia, J. M. 2004. Extreme Value and Related Models with Application in Engineering and Science. Wiley-Intercience.

[3] Davison, A. C., and Hinkley, D. V. 1997. Bootstrap Method and Their Application. Cambridge University Press.

[4] Cuong, D. K., Dam, D. T., and Duong, D. D. T. 2017. "Extreme Value Distributions in Hydrological Analysis of Some Area in the Mekong Delta. Some new Applications of Mathematical, especially to Econometrics." In Proceedings of the Second Vietnam International Applied Mathematics Conference VIAMC, 198-206.

[5] Dam, D. T., Tai, V. V., Truc, P. M., and Cuong, D. K. 2016. "Forecasting Crest of Salinity at the Main Stations of Ca Mau Province by Fuzzy Time Series Model.” Can Tho University Journal of Science 74: 86.

[6] Dam, D. T., Cuong, D. K., and Linh, H. M. 2017. "Solving the Problem of Hydrometeorological Data Analysis by Random Process Theory.” Science \& Technology Development Journal 20: 101-6.

[7] Efron, B., and Tibshirani, R. 1993. An Introduction to the Bootstrap. Chapman \& Hall/CRC.

[8] Mudelsee, M. 2014. Climate Time Series Analysis. Classical Statistical and Bootstrap Method, 2nd ed.

[9] Hall, P. 1992. The Bootstrap and Edgewood Expansion. Springer Series in Statistics.

[10] Radovanov, B., and Marcikie A. 2014. "A Comparison of Four Different Block Bootstrap Methods.” Croatian Operational Research Review 5: 189-202. 